\title{
Positive Effects of Acupuncture on Atopic Dermatitis
}

\author{
Rezan Akpinar ${ }^{*}$ and Saliha Karatay ${ }^{2}$ \\ ${ }^{1}$ Queensborough Community College, New York, USA \\ ${ }^{2}$ Physical Medicine and Rehabilitation, Ankara, Turkey
}

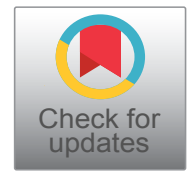

*Corresponding author: Rezan Akpinar, Queensborough Community College, New York, USA, Tel: 917-836-0187, E-mail: rakpinar@qcc.cuny.edu

\begin{abstract}
Atopic dermatitis, or allergic eczema, is a chronic inflammatory skin condition which is noncontagious and leads to itchy, dry skin lesions that can damage the intact skin barrier. Although the etiology is not known, it is widely believed that family history and allergies play a role. The incidence of atopic dermatitis is usually higher in early childhood, while other allergic conditions can develop later in life, such as allergic rhinitis and asthma. Atopic dermatitis is typically treated through topical ointments and the systematic use of pharmaceuticals to control symptoms. Long term or improper use of medications can lead to other complications. When symptoms of the condition are chronic or recurring, they can seriously impact one's quality of life, resulting in many patients seeking alternative care. However, the efficacy and safety of alternate modalities remain a major concern in the health care field. Acupuncture is one such modality used in the treatment of atopic dermatitis. Although multiple studies have established the safety of acupuncture, its efficacy with atopic dermatitis and similar conditions is still being explored. This review will discuss the use of various forms of acupuncture and their mechanisms in addressing atopic dermatitis.
\end{abstract}

\section{Keywords}

Atopic dermatitis, Treatment, Acupuncture

Atopic dermatitis is a chronic condition characterized by pruritus, dry skin, and eczema [1]. It is known for its propensity to relapse under certain conditions. Although the etiology is unknown, it is believed that genetic factors, together with family history of atopic disease, stress, allergies and inflammatory conditions play a role in the appearance of the condition. Immunological variations such as overproduction of the IgE, antigen, specific IgE antibodies, epidermal barrier dysfunction, and environmental triggers are identified in its pathogenesis $[2,3]$. Uncertainty of the etiology and pathogenesis, combined with the multiple factors that play a role in the disease process, impacts the probability for successful treatment. There is no single treatment protocol for atopic dermatitis.

Local and systemic pharmaceuticals, as well as nondrug substances, have traditionally been used to treat the condition. Treatment with topical corticosteroids, antibacterial agents, topical calcineurin inhibitors, antihistamines, immunosuppressives, emollients, wetwrap, phototherapy, biological treatment with monoclonal antibodies have all exhibited varying degrees of success in eliminating symptoms of atopic dermatitis $[3,4]$. But it is also true that these same treatment modalities have sometimes been ineffective, or have caused side effects. Uncontrolled symptoms, reoccurring flare-ups, and side effects of pharmaceuticals have incentivized patients to seek alternative treatment modalities. The number of patients with atopic dermatitis that use Complementary and Alternative Medicine (CAM) has increased [5]. According to statistics, $6 \%$ of patients with dermatological conditions in the United States use some form of CAM [6]. Acupuncture is becoming increasingly popular among atopic dermatitis patients $[7,8]$.

Acupuncture has been in use for the past 3,000 to 5,000 years [9]. It is one of the most universally recognized components of Traditional Chinese Medicine, or TCM. TCM also incorporates dietary therapies, herbal medicine, body work, and exercise. All components of TCM use basic principles such as Yin/Yang, Five Elements, Jing/Luo, and Zang/Fu organ theories as a foundation in the assessment of health for the purposes of

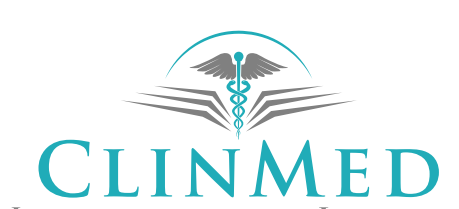

INTERNATIONAL LIBRARY

Citation: Akpinar R, Karatay S (2018) Positive Effects of Acupuncture on Atopic Dermatitis. Int J Aller Medications 4:030. doi.org/10.23937/2572-3308.1510030

Accepted: July 04, 2018: Published: July 06, 2018

Copyright: (c) 2018 Akpinar R, et al. This is an open-access article distributed under the terms of the Creative Commons Attribution License, which permits unrestricted use, distribution, and reproduction in any medium, provided the original author and source are credited. 
determining the best form of treatment [10]. The approach to treatment in this context is more patient-centered, where signs and symptoms are evaluated, and a pathological syndrome analysis is conducted with information gathered according to TCM principles. Treatment is geared toward the causative factor(s) of the underlying issue, not just symptom control.

One of the most debilitating symptoms of atopic dermatitis is severe pruritus [11]. Scratching of lesions due to pruritus can result in destruction of the natural skin barrier, leading to complications such as bleeding and infection. Therefore, most of the studies investigating the efficacy of acupuncture in atopic dermatitis have focused on pruritus. At first, antipruritic effects of acupuncture have been demonstrated with artificially-induced pruritus in healthy patients in placebo-controlled studies [12]. In addition, a number of reports supports the antipruritic effects of acupuncture in diseases that cause pruritus [13-15]. In comparative studies, acupuncture has been shown to provide better results than antihistamines and preventive antipruritic systemic drugs [12,16]. Although studies demonstrate positive outcomes, the mechanism of action with the antipruritic effects of acupuncture is still unknown. It is believed that, as with other effects of acupuncture, antipruritic effects are due to peripheral and central mechanisms. Anti-allergic and anti-inflammatory effects might be the reasons behind peripheral antipruritic actions. It is known that IgE expression plays an important role in acute and chronic skin inflammation [14]. IgE is carried on basophils cells' surfaces, which is important in IgE-mediated allergies. A pilot study showed that acupuncture caused a reduction in activation of basophils in patients with atopic dermatitis [15]. In an animal study, acupuncture reduced serum IgE levels, as well as mRNA expressions of IL-4, IL-8, and TNF- $\alpha$ pro-inflammatory cytokines [14]. In addition, decreasing in mast cell count and histamine release could also play a role in alleviation of the lesions and antipruritic effects of acupuncture in atopic dermatitis patients [17]. Modulatory effects of acupuncture on neurotransmitters, cytokines, hormones, growth factors, and oxidative factors have been known for a long time [8]. Acupuncture is used in inflammatory diseases due to its effect in reducing pro-inflammatory cytokines, cyclooxygenase-2, and nitric oxide synthesis $[18,19]$. The anti-inflammatory effects of acupuncture could also contribute to the treatment of atopic dermatitis. Increase in blood flow to local needle insertion sites, as well as the structures that are related to the area, have been demonstrated in previous studies $[8,20]$. These factors could be responsible for healing lesions, as well as normalizing of the thickening of the skin.

Other than the peripheral effects of acupuncture on atopic dermatitis, there could be central mechanisms as well. The mechanism of the antipruritic effects of acupuncture in the brain has been studied with functional Magnetic Resonance Imaging (fMRI). Napadow, et al. have observed brain activities in the validated temperature-modulation itch model with $\mathrm{fMRI}$ on patients with atopic dermatitis [12]. In this study, patients were placed into four separate groups while their brain activities were recorded through fMRI. The four groups consisted of verum (real) acupuncture, placebo acupuncture, verum antihistamine solution, and placebo solution. Researchers reported a decline in the itch-evoked activation in insula, putamen, the premotor cortex, and the prefrontal cortex of the brain, as well as a decrease in itch sensation in acupuncture group patients with atopic dermatitis. These were not observed in antihistaminic and placebo groups. Itch reduction was also correlated with the level of reduction in response in putamen. Due to these results, researchers concluded that putamen might be responsible for the itch sensation in atopic dermatitis patients. Previous research has already established the role of central sensitization of itch in diseases that caused chronic itch, such as atopic dermatitis [21]. Acupuncture treatment can be effective in modulation of central sensitization.

In addition to addressing central and peripheral anti-itch effect as discussed above, acupuncture can also affect stress factors which are important in etiopathogenesis of atopic dermatitis. Acupuncture is an effective modality for treating emotional disturbances and for reducing stress responses. Therefore, it has been used in the treatment of depression and anxiety disorders [22]. Research has shown that acupuncture can induce beneficial changes in the levels of neurotransmitters like serotonin, substance-P, endorphins, and enkephalins in patients with pain and emotional disturbances [23]. Acupuncture has been found to affect the limbic system, which is responsible for stress-related symptoms [24]. The role of stress is important in the etiology of allergy-related skin disorders and their propensity for reoccurrence. Therefore, acupuncture is also valuable in the treatment of atopic dermatitis by virtue of the fact that it can reduce a patient's level of stress.

Various systemic reviews and a meta-analysis have assessed studies about the efficacy of acupuncture in the treatment of atopic dermatitis $[7,25,26]$. The various Randomized Controlled Trials (RCTs) that met the inclusion criteria demonstrated a reduction in itching, lesion size, and recurrence in an acupuncture group compared to a control group. While there were no statistical differences between control and acupuncture groups in some of the studies, there were others that did not use control groups. All systemic reviews and the meta-analysis concluded that there is a need for high quality, standardized, and controlled RCTs. TCM treatment philosophy might be the reason behind the variances in the study results. According to TCM, the disease process in general is the result of an imbalance in one of the five vital substances, or energy flow in the channel system. In terms of a five-element theory, this is also seen as an imbalance between the five elements. 
Causative factors in imbalances can vary from patient to patient. Therefore, different patients who are experiencing the same disease process might exhibit different underlying causative factors. In these cases, when a study uses standardized point protocols, the efficacy of acupuncture can vary, which challenges RCT results as well as diminishing the effectiveness of acupuncture. To overcome this issue, some RCTs used acupoints that addressed the individualized needs of patients in addition to standardized protocols that have been shown to be effective [15]. There are considerable published case studies that show successful results using syndrome analysis and individualized acupuncture treatments.

Large numbers of studies conducted on patients with pruritus and dermatological conditions such as atopic dermatitis used LI 11 (Quchi) as a standard antipruritic point. There are multiple studies showing reduction in itch sensation through the stimulation of this point with needling, pressure, moxa, or cold applications [14,27]. LI 11 is an important point on the Large Intestine channel, this point is used to clear heat, which is considered a yang pathogen, from the skin (which happens to be the Yang part of the body). Because LI 11 is on a Yang Ming channel, it is more effective in clearing heat, which is why it used in cases of itchy, dry, and scaly inflamed skin conditions. Quchi is located at the lateral end of the transverse cubital crease when the arm is flexed ninety degrees [28].

In other studies, local points were used instead of these standard points. Park, et al. have investigated differences in responses between manipulation of $\mathrm{LI} 11$ and local points [14]. According to this study, both local points and $\mathrm{LI} 11$ reduced cutaneous hyperplasia and serum IgE levels, but only LI 11 was able to reduce mRNA expression of pro-inflammatory cytokines and proteins.

Other than TCM acupuncture, there are many other styles of acupuncture that address dermatological diseases. Auricular acupuncture, which is a component of microsystem has been used in treatment of dermatological conditions. Microsystems are parts of the body that represent the whole body. Utilizing principles similar to those in reflexology, scalp, ears, eyes, tongue, and other locations in body are used for diagnostic and treatment purposes [29]. Allergy points exist in many different styles of ear acupuncture. A study conducted by Soliman compared Dr. Poul Nogier's auricular protocol for allergies with Soliman's Auricular Allergy Treatment (SAAT) [30]. Dr. Nogier, who developed auricular microsystems in the 1960 s, used an antihistaminic point to treat allergies. Dr. Nogier's antihistaminic point is located in the apex of the ear, where the external and internal surfaces meet [31]. SAAT is an expansion of this system, which locates active points in the ear during an allergic attack. Point stimulation is performed using intradermal needles, which stay in the dermal layer without harming cartilage tissue. Both studies resulted in elimination of the allergies, but the time for relapse was shorter with Dr. Nogier's treatment protocol. In both cases, patients only received auricular therapy.

There are multiple case studies which incorporated different styles of acupuncture with successful results. One of them is Sa-Am acupuncture, which is a Korean style. A case study presented by Jeon, et al. used Korean Sa-Am acupuncture to treat a 22-year-old female patient with an eight-year history of atopic dermatitis [32]. Her first visit to the office was during an acute flare-up, which was accompanied by erythematous skin rash. Syndrome analysis revealed that the patient was not in balance between the Arm Tai Yin channel and Foot Tai Yang channel. Three points SP 3, LU 9, and BL 67, were manipulated to balance these channels. Pictorial progression of the treatment revealed disappearance of the lesion. In another case study from Korea, Lee, et al. treated a 25-year-old male patient with a history of atopic dermatitis, who was hospitalized due to Kaposi's Varicella eruption [33]. Eruptions were cleared within ten days, with systemic and local application of herbal formulas and the use of Master Tung's points.

Female patients with atopic dermatitis can experience flare-ups during pregnancy. Because the use of certain pharmaceutical drugs during pregnancy is not recommended [34], acupuncture can offer a safe and reliable treatment method for pregnant women suffering from atopic dermatitis. However, practitioners must keep in mind that there are specific points which should not be used on pregnant patients. Another case report presented successful results in treatment of atopic dermatitis with Korean acupuncture in pregnant women [35]. Three pregnant women who could not use conventional medicine were treated with acupuncture, Korean herbal medicine, and herbal wet wraps. In these instances, local points near the lesions were needled as well as standard acupuncture treatments. Severity Scoring Atopic Dermatitis (SCORAD) index showed improvement in the severity of atopic dermatitis and relief of its symptoms. There were no undesirable side effects on the pregnancy, including fetal or neonatal development. This was confirmed by laboratory tests and observational monitoring.

Other than safe use of acupuncture in pregnant women, case reports demonstrate that acupuncture is used with pediatric atopic dermatitis patients. Yu and Kizhakke Veettil worked on two pediatric patients, ages 8 and 11 , who were diagnosed with atopic dermatitis at infancy [1]. Patients were treated with TCM style acupuncture. Syndrome analysis for both conditions revealed a deficiency state which was rectified by using strengthening points. Points included in the study were CV 12, ST 36, SJ 5, LI 11, LU7, ST 40, SP 9 and KI 3. After a full course of 12 treatment sessions, lesions and pruritus completely disappeared. In this case study, patients were checked at regular intervals and no reoccurrence 
was reported for up to 18 months post-treatment.

There is no universally recognized "gold standard" treatment protocol for atopic dermatitis. This is largely due to the myriad of factors that play a role in its etiology. Varying degrees of success have been reported with pharmaceutical and non-pharmaceutical treatment methods. The availability of clinical evidence behind many of these treatments is very low due to the insufficient number of controlled RCTs [4]. Side effects that develop due to the use of conventional management and unresponsiveness to medication are limiting factors behind the use of treatments for prolonged periods of time. Taking these issues into consideration, acupuncture which improves symptoms of atopic dermatitis and prolongs the duration between flare-ups without serious side effects could be promising for the patient population at large.

\section{References}

1. Yu J, KizhakkeVeettil A (2011) Acupuncture for the management of pediatric atopic dermatitis: Case reports. Medical Acupuncture 23: 53-56.

2. Jung $T$ (2002) New Treatments for Atopic Dermatitis. Clin Exp Allergy 32: 347-354.

3. Pelc J, Czarnecka-Operacz M, Adamski Z (2018) The structure and function of the epidermal barrier in patients with atopic dermatitis-treatment options, part two. Postepy Dermatol Alergol 35: 123-127.

4. Alomar A, Yélamos O (2013) Guidelines review on atopic dermatitis management. Clin Pract 10: 311-316.

5. Boneberger S, Rupec RA, Ruzicka T (2010) Complementary therapy for atopic dermatitis and other allergic skin diseases: Facts and controversies. Clin Dermatol 28: 57-61.

6. Smith N, Shin DB, Brauer JA, Mao J, Gelfand JM (2009) Use of complementary and alternative medicine among adults with skin disease: Results from a national survey. $J$ Am Acad Dermatol 60: 419-425.

7. Ma C, Sivamani RK (2015) Acupuncture as a Treatment Modality in Dermatology: A Systematic Review. J Altern Complement Med 21: 520-529.

8. Van den Berg-Wolf M, Burgoon T (2017) Acupuncture and cutaneous medicine: Is it effective? Med Acupunct 29: 269-275.

9. Giovanni Maciocia (2015) Foundations of chinese medicine-A comprehensive text. ( $3^{\text {rd }}$ edn), Elsevier Health Sciences.

10. Swati K, Dasgupta A, Mitra A (2011) Treatment efficacy of acupuncture, yoga and homeopathy in the indian context: A review IJBSAHM 2: 1-20.

11. Chi Yu, Pei Zhang, Zheng-Tao Lv, Jing-Jing Li, Hong-Ping $\mathrm{Li}$, et al. (2015) Efficacy of acupuncture in itch: A systematic review and meta-analysis of clinical randomized controlled trials. Evid Based Complement Alternat Med 2015: 208690.

12. Napadow V, Li A, Loggia ML, Kim J, Schalock PC, et al. (2014) The brain circuitry mediating antipruritic effects of acupuncture. Cereb Cortex 24: 873-882.

13. Pfab F, Kirchner MT, Huss-Marp J, Schuster T, Schalock PC, et al. (2012) Acupuncture compared with oral antihistamine for type i hypersensitivity itch and skin response in adults with atopic dermatitis: A patient and examiner - blind- ed, randomized, placebo-controlled, Crossover Trial. Allergy 67: 566-573.

14. Park JY, Park HJ, Choi YY, Kim MH, Kim SN, et al. (2013) Effects of acupuncture on 1-chloro-2,4-dinitrochlorobenzene-induced atopic dermatitis. Evid Based Complement Alternat Med 2013: 982095.

15. Pfab F, Athanasiadis GI, Huss-Marp J, Fuqin J, Heuser B, et al. (2011) Effect of acupuncture on allergen-induced basophil activation in patients with atopic eczema: A pilot trial. J Altern Complement Med 17: 309-314.

16. Ikoma A, Steinhoff M, Ständer S, Yosipovitch G, Schmelz M (2006) The neurobiology of itch. Nat Rev Neurosci 7: 535-547.

17. Pfab F, Huss-Marp J, Gatti A, Fuqin J, Athanasiadis GI, et al. (2010) Influence of acupuncture on type i hypersensitivity itch and the wheal and flare response in adults with atopic eczema - a blinded, randomized, placebo-controlled crossover trial. Allergy 65: 903-910.

18. Mc Donald JL, Cripps AW, Smith PK, Smith CA, Xue CC, et al. (2013) The anti-inflammatory effects of acupuncture and their relevance to allergic rhinitis: A narrative review and proposed model. Evid Based Complement Alternate Med 2013: 591796.

19. Lee JH, Jang KJ, Lee YT, Choi YH, Choi BT (2006) Electroacupuncture inhibits inflammatory edema and hyperalgesia through regulation of cyclooxygenase synthesis in both peripheral and central nociceptive sites. Am J Chin Med 34: 981-988.

20. Cakmak YO, Akpinar IN, Ekinci G, Bekiroglu N (2008) Point and frequency-specific response of the testicular artery to abdominal electroacupuncture in humans. Fertil Steril 90: 1732-1738.

21. Ikoma A, Fartasch M, Heyer G, Miyachi Y, Handwerker $\mathrm{H}$, et al. (2004) Painful stimuli evoke itch in patients with chronic pruritus: Central sensitization for itch. Neurology 62: 212-217.

22. Asher GN, Gerkin J, Gaynes BN (2017) Complementary therapies for mental health disorders. Med Clin North Am 101: 847-864.

23. Karatay S, Okur SC, Uzkeser H, Yildirim K, Akcay F (2018) Effects of acupuncture treatment on fibromyalgia symptoms, serotonin, and substance $p$ levels: A randomized sham and placebo-controlled clinical trial. Pain Med 19: 615-628.

24. Hui KK, Marina O, Liu J, Rosen BR, Kwong KK (2010) Acupuncture, the limbic system, and the anticorrelated networks of the brain. Auton Neurosci 157: 81-90.

25. Tan HY, Lenon GB, Zhang AL, Xue CC (2015) Efficacy of acupuncture in the management of atopic dermatitis: A systematic review. Clin Exp Dermatol 40: 711-715.

26. Shi ZF, Song TB, Xie J, Yan YQ, Du YP (2017) The traditional chinese medicine and relevant treatment for the efficacy and safety of atopic dermatitis: A systematic review and meta-analysis of randomized controlled trials. Evid Based Complement Alternat Med 2017: 6026434.

27. Tsai KS, Chen YH, Chen HY, Shen EY, Lee YC, et al. (2014) Antipruritic effect of cold stimulation at the quchi acupoint (li11) in mice. BMC Complement Altern Med 14: 341.

28. Deadman P, Al-Khafaji M, Baker K (2007) A manual of acupuncture. ( ${ }^{\text {nd }}$ edn), Journal of Chinese Medicine Publications.

29. Oleson T (2003) Auriculotherapy manual chinese and western systems of ear acupuncture. ( $3^{\text {rd }}$ edn), Elsevier Limited. 
30. Soliman N (2014) A comparison study of the effectiveness of SAAT (Soliman's Auricular Allergy Treatment) approach and nogier's allergy treatment technique. Medical Acupuncture 26: 167-172.

31. Nogier R (2009) Auriculotherapy. ( $1^{\text {st }}$ edn), Thieme Paperback.

32. Jeon YC, Lee HB (2016) Treatment of an adult patient with atopic dermatitis using traditional korean medicine, especially sa-am acupuncture. J Acupunct Meridian Stud 9: 322-324.
33. Lee DJ, Kwon K, Sun SH, Seo HS (2014) Kaposi's varicelliform eruption in atopic dermatitis treated with korean medicine. J Pharmacopuncture 17: 80-83.

34. Babalola O, Strober BE (2013) Treatment of atopic dermatitis in pregnancy. Dermatol Ther 26: 293-301.

35. Kim M, Yun Y, Kim Ks, Choi I (2013) Three cases of atopic dermatitis in pregnant women successfully treated with korean medicine. Complement Ther Med 21: 512-516. 